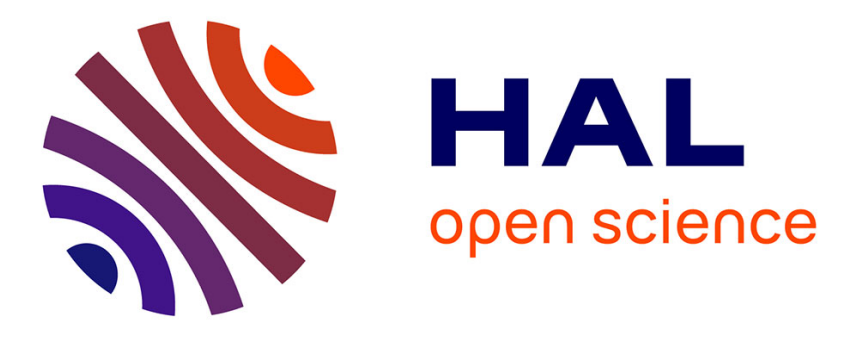

\title{
On phase locking phenomena in Kerr combs
}

Aurélien Coillet, Irina Balakireva, Rémi Henriet, Laurent Larger, Yanne

Kouomou Chembo

\section{To cite this version:}

Aurélien Coillet, Irina Balakireva, Rémi Henriet, Laurent Larger, Yanne Kouomou Chembo. On phase locking phenomena in Kerr combs. Proceedings 8600 of SPIE Conference on Laser Resonators, Microresonators, and Beam Control XV, Jan 2013, United States. pp.86000C. hal-00914643

\section{HAL Id: hal-00914643 \\ https://hal.science/hal-00914643}

Submitted on 5 Dec 2013

HAL is a multi-disciplinary open access archive for the deposit and dissemination of scientific research documents, whether they are published or not. The documents may come from teaching and research institutions in France or abroad, or from public or private research centers.
L'archive ouverte pluridisciplinaire HAL, est destinée au dépôt et à la diffusion de documents scientifiques de niveau recherche, publiés ou non, émanant des établissements d'enseignement et de recherche français ou étrangers, des laboratoires publics ou privés. 


\title{
On phase locking phenomena in Kerr combs
}

\author{
Aurélien Coillet, Irina Balakireva, Rémi Henriet, Laurent Larger and Yanne Chembo \\ FEMTO-ST Institute, UMR 6174 CNRS - Université de Franche-Comté, 32 avenue de \\ l'observatoire 25044 Besançon Cedex
}

\begin{abstract}
Based on a modal description of the evolution Kerr combs in a whispering-gallery mode resonator, we numerically investigate the phase behavior of the different spectral lines of the spectrum. We show that a stable phase relation exist between adjacent modes in primary combs. This result is of great interest for metrological applications were one phase noise is an issue. For high input power however, chaotic signals are observed.
\end{abstract}

Keywords: Kerr frequency combs, Whispering-gallery mode resonators, Nonlinear dynamics

\section{INTRODUCTION}

Optical frequency combs are sets of regularly spaced spectral lines in the optic frequency range. They are usually generated from mode-locked femtosecond laser, since a periodic train of pulses yield such equidistant lines in the spectral domain. Those combs have many applications in time-frequency metrology, spectroscopy, navigation systems, sensing $[1,2]$... A new way of generating these frequency combs has recently been demonstrated and it relies on the optical Kerr effect in ultra-high-Q whispering-gallery mode (WGM) resonators [3]. In such resonators, the resonant light is enhanced and nonlinear effects can occur at low input powers. In particular, four-wave mixing (FWM) allows for the creation and mixing of frequencies under strict laws of energy and momentum conservation. Only frequency lines separated by the free spectral range (FSR) of the resonator can exist, and a Kerr comb is finally created.

The modeling of this process is however quite complex, since many interactions can occur between modes through the $\chi^{(3)}$ susceptibility of the medium. A few works have been published on this topic [4,5], and a recent work using a modal approach has been proposed [6]. In this work, we use this model to perform numerical simulations and study the mechanism allowing phase locking during the growth of the Kerr comb.

\section{SYSTEM UNDER STUDY}

The typical scheme of comb generator in a WGM resonator is presented in Figure 1. A continuous-wave laser is amplified and coupled to a WGM resonator thanks to a tapered fiber. In a fiber with such a diameter $(\sim 3 \mu \mathrm{m})$, an important fraction of the propagating mode is located in the air, as an evanescent field. By bringing the fiber close to the resonator, light can be coupled inside the WGM resonator. The light at the output of the fiber is monitored either in the spectral domain with a spectrum analyzer, either in the temporal domain with an oscilloscope.

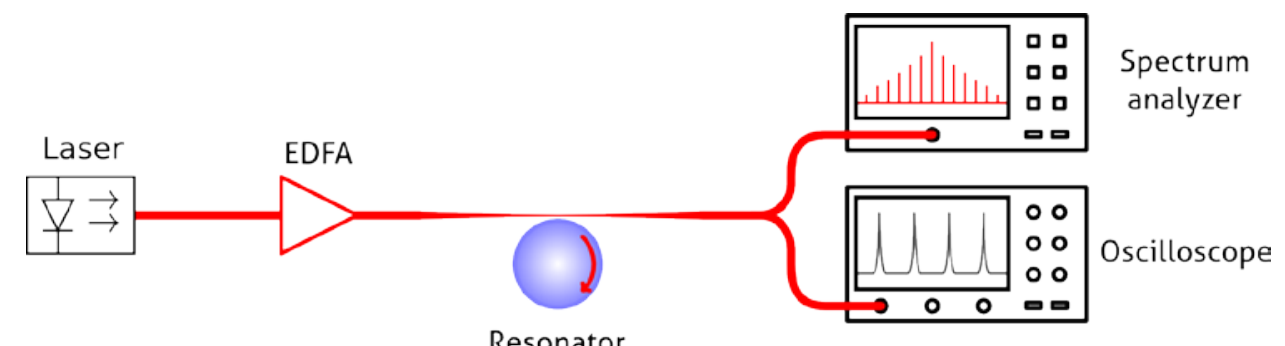

Resonator

Figure 1. Experimental setup of the Kerr combs generation in a WGM resonator. A continuous laser is amplified and coupled to a WGM resonator using a tapered fiber. The output is either acquired with a spectrum analyzer or an oscilloscope.

The size of the WGM resonator is chosen so that its FSR is in the wanted frequency range. In our case, we will focus on millimeter-sized resonators for a 10-GHz range FSR. The bulk medium of the resonator can be amorphous (e. g. fused 
silica) or crystalline. The latter requires a symmetry inversion for the Kerr nonlinearity to be significant. This is the case in $\mathrm{CaF}_{2}$ an $\mathrm{MgF}_{2}$ crystals, which are characterized by very low absorption losses, leading to potential ultra-high Qfactors. Our study takes the example of a 5-mm diameter $\mathrm{CaF}_{2}(\mathrm{n}=1.43$ at $1550 \mathrm{~nm})$ resonator, with a $13.4 \mathrm{GHz}$ FSR and a quality factor of $3.10^{9}$.

\section{MODAL DESCRIPTION}

The modal description proposed in [6] starts from the Maxwell equation and derives the temporal evolution of each resonant mode in the cavity. It takes into account both the FWM gain and the dispersion of the cavity. Using a slowly varying amplitude expansion, the rate equations describing the evolution of the envelope $A_{\eta}$ of each mode is obtained:

$$
\dot{A}_{\eta}=-\frac{1}{2} \Delta \omega_{\eta} A_{\eta}-i g_{0} \sum_{\alpha, \beta, \mu} \Lambda_{\eta}^{\alpha \beta \mu} A_{\alpha} A_{\beta}^{*} A_{\mu} e^{i \varpi_{\alpha \beta \mu \eta} t}+\frac{1}{2} \Delta \omega_{\eta} F_{\eta} e^{i\left(\Omega_{0}-\omega_{\eta}\right) t}
$$

The modal bandwidth $\Delta \omega_{\eta}$ corresponds to the inverse of the modal photon lifetime. $g_{0}$ stands for the FWM gain while $\Lambda_{\eta}^{\alpha \beta \mu}$ is the intermodal coupling factor between the four interacting modes. The resonator's dispersion is taken into account in the $\varpi_{\alpha \beta \mu \eta}=\omega_{\alpha}-\omega_{\beta}+\omega_{\mu}-\omega_{\eta}$ parameter, since it would be null in the case of perfectly equidistant spectral lines. Finally, the last term corresponds to the external pumping of the cavity, with amplitude $F_{\eta}$ and angular frequency $\Omega_{0}$.
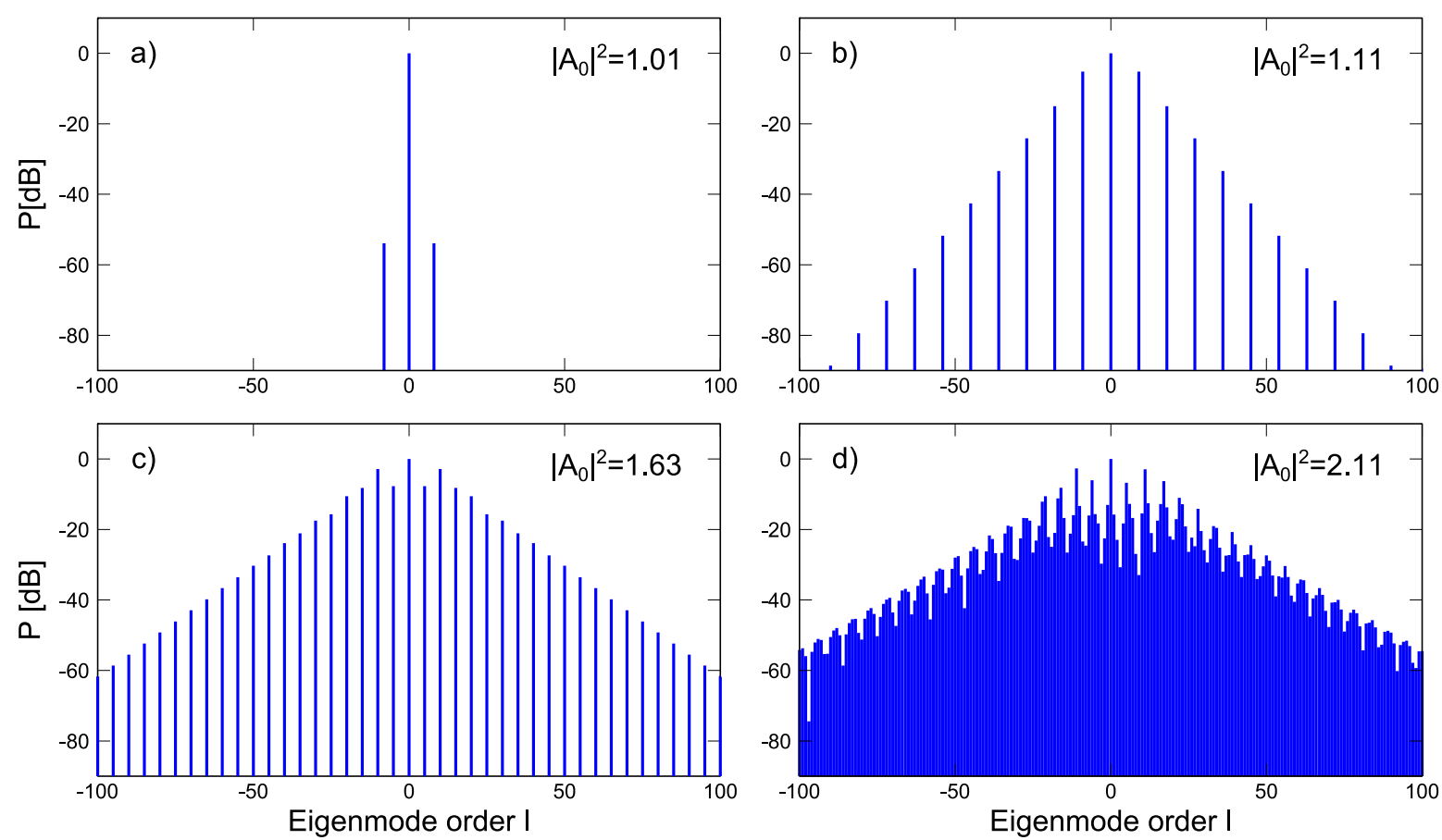

Figure 2. Simulated combs using the modal description for various pumping power. a) Just above threshold, only a couple of side lines are excited, separated from the central line by 9 FSR. b) At higher excitation, a primary comb is fully formed. c) When still increasing the pump power, a secondary comb appears, making the interval between adjacent spectral lines half of the one of the primary comb. d) Finally, at high power, each resonant mode is populated.

These equations can be numerically solved with a fourth-order Runge-Kutta algorithm. In the following simulations, 201 modes were studied, corresponding to a $\sim 20 \mathrm{~nm}$ wavelength span. Each mode is initialized with a random complex amplitude taken in a Gaussian distribution with mean value and standard deviation equal to 10, corresponding to an 
average of 100 photons in each mode, since $\left|A_{\eta}\right|^{2}$ is the number of photon in the $\eta^{\text {th }}$ mode. Simulations are performed for a total duration of $2 \mathrm{~ms}$, such that the steady state, if any, is reached. The combs displayed in Figure 2 correspond to various pumping amplitude expressed in $\left|A_{0}^{t h}\right|^{2}$ units, where $\left|A_{0}^{t h}\right|^{2}$ is the threshold number of photons in the pumping mode. The parameters used in these simulations are $\Omega_{0}-\omega_{0}=0$ (the pump laser is perfectly resonant), the dispersion term $\varpi_{\alpha \beta \mu \eta}=\frac{1}{2} \zeta\left(l_{\alpha}^{2}-l_{\beta}^{2}+l_{\mu}^{2}-l_{\eta}^{2}\right)$ where $l_{\alpha}^{2}$ corresponds to the $\alpha^{\text {th }}$ mode index and the parameter $\zeta=11800$ rad corresponds to a small, anomalous dispersion. As shown in [6], the presented computer-simulated combs are in good agreement with experimental results already obtained.

\section{PHASE EVOLUTION AND LOCKING}

The numerical simulation of the model allows us to follow the evolution of the phase of each spectral line separately. However, the relative phases of the different modes with the pump mode are not trivial, and, according to our model's equation, we should study the following quantity:

$$
\arg \left(\frac{A_{\eta} e^{i \varpi_{0 \eta} t}}{A_{0}}\right)
$$

This argument compares the phases of the $\eta^{\text {th }}$ mode and the pump mode and takes into account the effect of the dispersion. If this quantity tends to a finite value, the two modes will be phase-locked.

In Figure 3, we present the results for WGM resonator excited at $1.4\left|A_{0}^{t h}\right|^{2}$, leading to a primary comb. The relative phase of each excited mode and the pump mode is plotted as a function of time. At this pump power, the interval between each mode is of 9 FSR, and the dispersion compensation was adjusted accordingly.

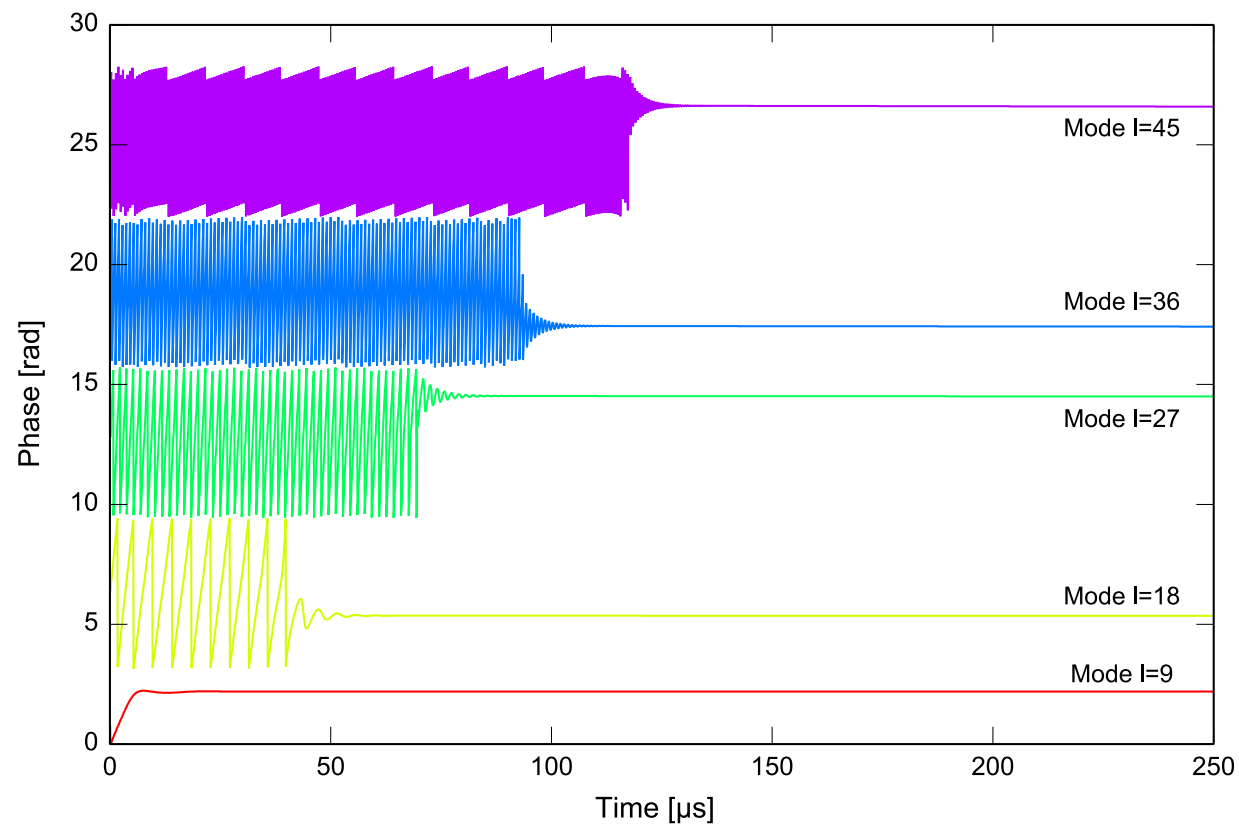

Figure 3. Evolution of the corrected phase between the 5 first excited modes, mode 9, 18, 27, 36 and 45 . The y-scale was shifted by $2 \pi$ for each mode, for a better visibility of the figure. The $9^{\text {th }}$ mode is the first appearing and its phase is locked to the pump since the very beginning. On the contrary, the higher order mode are locked successively to the pump.

The resulting curves show that the 9th mode, that is the first excited mode, is very quickly phase locked. The following modes are phase-locked only after, and the farther the later. Before being phase locked, the different modes oscillate freely at their natural frequency. 
For higher pump power, a secondary comb appears for a pump power of $1.63\left|A_{0}^{t h}\right|^{2}$, as shown on Figure 2. The relative phase of the modes is plotted on Figure 4 . As in the previous case, the primary comb (modes $10,20, \ldots$ ) is still quickly phase-locked to the pump. However, this secondary mode does not follow the same relationship and oscillate at its own frequency.

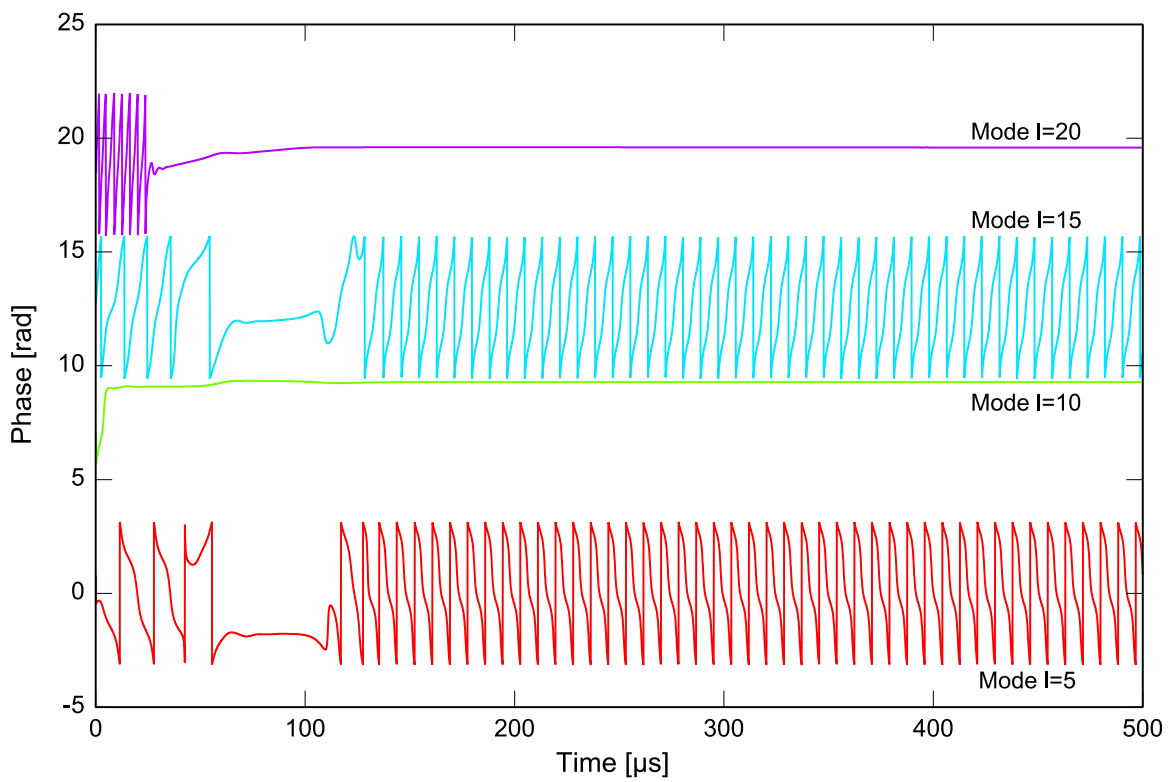

Figure 3. Evolution of the relative phase for a secondary comb, at $1.63\left|A_{0}^{\text {th }}\right|^{2}$ pump power. The primary comb is still phaselocked, but the secondary comb does not seem to obey any relationship with the pump mode.

Still increasing the power, at 2.1 $\left|A_{0}^{\text {th }}\right|^{2}$, the generated comb displays now chaotic behavior, and the phase no longer follows the pump mode. A similar pattern to the previous case is still visible, where the primary comb does not show fast and brutal changes in phase while in-between modes do. Actually, the farther the mode under study is from one mode of the primary comb, the faster its phase drifts.

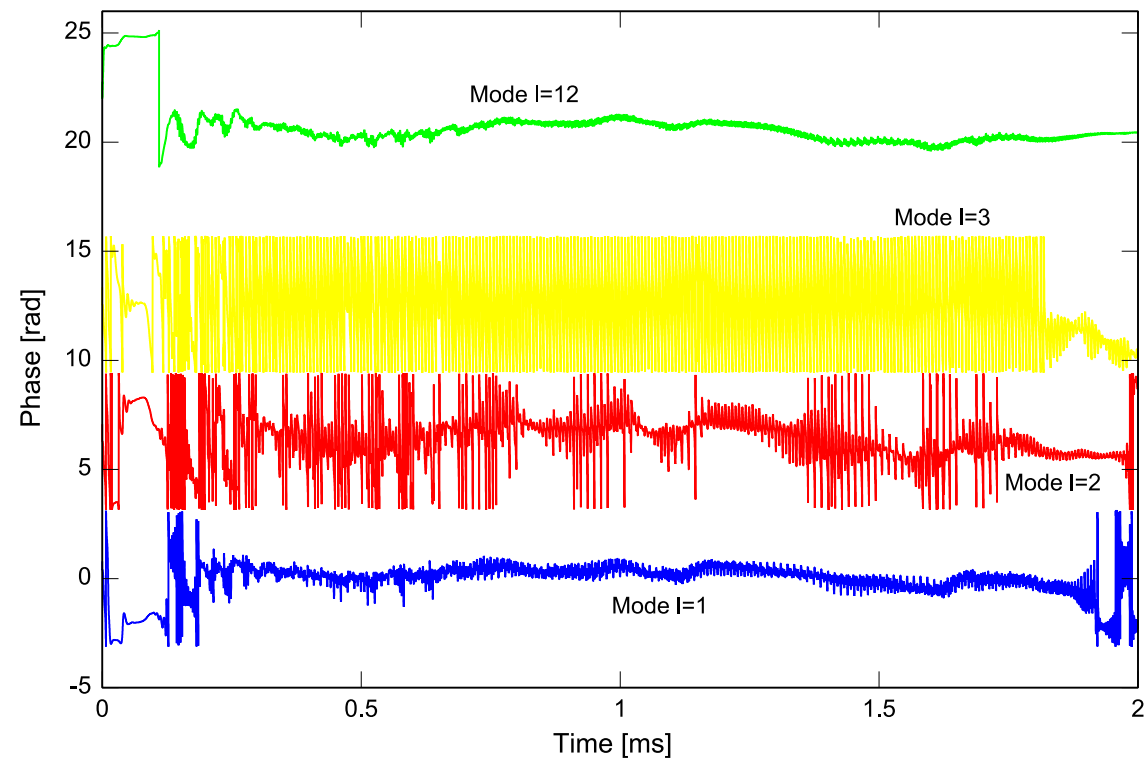

Figure 3. Evolution of the relative phase for a chaotic comb, at 2.11 $\left|A_{0}^{t h}\right|^{2}$ pump power. The phase locking shows signs of chaotic behavior, yet the changes are less brutal for modes next to a primary comb mode. 


\section{CONCLUSION}

In this work, we studied the evolution of the phase of the mode relatively to the central mode. This study must take into account the dispersion-induced drift, varying in $l^{2}$. Using a Runge-Kutta approach we show that phase-locking between each mode of the primary comb occurs while the comb is being generated. This results confirm the metrological interest for such combs. When the secondary comb appears, however, the new spectral lines do not follow this pattern.

Increasing the pump power leads to a chaotic behavior of the phase, yet the phase drift is smaller for modes near or on the primary comb.

\section{ACKNOWLEDGMENTS}

Y. C. K. acknowledges financial support from the European Research Council through the project NexPhase (ERC StG 278616). Authors also acknowledge support from the Centre National de la Recherche Spatiale (CNES, France) through the project SHYRO (Action R\&T R-S10/LN-0001-004/DA:10076201), drom the ANR project ORA (BLAN 031202) and from the Région Franch-Comté, France.

\section{REFERENCES}

[1] Theodor W. Hänsch, “Nobel Lecture: Passion for precision,” Reviews of Modern Physics 781297 (2006).

[2] Steven T. Cundiff and Jun Ye, "Femtosecond optical frequency combs," Reviews of Modern Physics 75325 (2003).

[3] P. Del'Haye, A. Schliesser, O. Arcizet, T. Wilken, R. Holzwart and T. J. Kippenberg, “Optical frequency comb generation from a monolithic microresonator," Nature 4501214 (2007).

[4] Imad H. Agha, Yoshitomo Okawachi, Mark A. Foster, Jay E. Sharping, and Alexander L. Gaeta "Four-wavemixing parametric oscillations in dispersion-compensated high-Q silica microspheres," Phys. Rev. A 76043837 (2007)

[5] Andrey B. Matsko, Anatoliy A. Savchenkov, Dmitry Strekalov, Vladimir S. Ilchenko, and Lute Maleki, "Optical hyperparametric oscillations in a whispering-gallery-mode resonator: Threshold and phase diffusion," Phys. Rev. A 71033804 (2005)

[6] Yanne K. Chembo and Nan Yu, "Modal expansion approach to optical-frequency-comb generation with monolithic whispering-gallery-mode resonators," Physical Review A 82033801 (2010).

[7] Yanne K. Chembo, Dmitry V. Strekalov, and Nan Yu "Spectrum and Dynamics of Optical Frequency Combs Generated with Monolithic Whispering Gallery Mode Resonators,” Phys. Rev. Lett. 104103902 (2010) 